\title{
Histopathological examination of two cases of anterior staphyloma associated with Peters' anomaly and persistent hyperplastic primary vitreous
}

Akihisa Matsubara, Hironori Ozeki, Noriko Matsunaga, Miho Nozaki, Masayuki Ashikari, Shoichiro Shirai, Yuichiro Ogura

\begin{abstract}
Aims-To clarify the developmental mechanism and critical period for the uncommon complex of Peters' anomaly and persistent hyperplastic primary vitreous (PHPV).

Methods-Two eyes with Peters' anomaly and PHPV were histologically examined by serial section. One eye was enucleated at age 7 months (case 1) and the other at age 4 months (case 2) owing to severe anterior staphyloma.

Results-In both eyes, defects in the endothelium, Descemet's membrane, and posterior stroma were observed in the central cornea, and the degenerative lens adhered to the posterior surface of the defective corneal stroma. Also, in both eyes, the anterior chamber space was not formed and the undifferentiated iris stroma adhered to the posterior surface of the peripheral cornea. Mesenchymal tissue containing melanocytes was observed behind the degenerative lens, and the pigment epithelium was absent at the lower nasal side of the ciliary body in case 1 . In case 2, mesenchymal tissue containing scattered melanocytes in the vitreous cavity was seen on the posterior retina. Based on the histological findings, both cases were diagnosed as Peters' anomaly caused by the faulty separation of the lens vesicle, PHPV, maldevelopment of the iris and ciliary body, and goniodysgenesis.

Conclusion-Migratory disorders of neural crest cells from 4 to 7 weeks of gestation may be responsible for various ocular anomalies including Peters' anomaly and PHPV, as observed in these cases.
\end{abstract}

(Br f Ophthalmol 2001;85:1421-1425)

In 1906, Peters ${ }^{1}$ first reported on patients with central corneal opacity and ring-shaped iridocorneal adhesion caused by the absence of the corneal Descemet's membrane. Later, similar patients with congenital central corneal opacity due to the absence of the corneal endothelium, Descemet's membrane, and posterior stroma were diagnosed as having Peters' anomaly. ${ }^{2-4}$ Peters' anomaly is usually seen as an isolated ocular defect; however, the disease can accompany other ocular and systemic anomalies. ${ }^{5-9}$
Persistent hyperplastic primary vitreous (PHPV), first identified by Reese, ${ }^{10}$ is a congenital malformation of the primary vitreous that is characterised by a retrolental white plaque of fibrovascular tissue. Subsequently, Pruett and Schepen ${ }^{11}$ classified malformations involving a retrolental mass, as described by Reese, ${ }^{10}$ as anterior PHPV, and malformations involving a congenital retinal fold ${ }^{12}$ or ablatio falciformis congenita ${ }^{13}$ as posterior PHPV. In cases with anterior PHPV, the ciliary processes are drawn inward by their attachment to the white plaque, often lying against the posterior lens, so they are visible in the pupil, while, in cases with posterior PHPV, strands of glial tissue extending from the retina into the vitreous represent another characteristic histological finding. ${ }^{14}$ A variety of clinical findings can be associated with PHPV, including microphthalmos, glaucoma due to closure of the chamber angle, shallowing of the anterior chamber, corneal opacity, cataract, uveal coloboma, and retinal degeneration; however, PHPV is also often seen as an isolated defect. ${ }^{15}$

As described, both Peters' anomaly and PHPV were usually seen as isolated ocular diseases. Although the association of Peters' anomaly and PHPV was reported clinically ${ }^{8}$ and histopathologically, ${ }^{9}$ the developmental mechanism and critical period for the clinically uncommon complex of these two malformations has not been adequately explained embryologically to date. We recently examined two cases with the complex of these two malformations. In the present study, we examined histopathologically the enucleated eyeballs with various ocular anomalies, including Peters' anomaly and PHPV, by serial section, evaluated their histological findings embryologically and, finally, inferred their developmental mechanism and critical period.

\section{Patients and methods} CLINICAL COURSES

Case 1

A 6 day old boy was referred to our clinic with a white pupil in the left eye that was present at birth. At the first examination, a central corneal opacity, elongated ciliary processes, and whitish mass behind the lens were observed (Fig 1A). The anterior chamber was not formed, and the fundus could not be observed in the left eye. No pathological findings were seen in the right eye. Intraocular pressure (IOP) was $8 \mathrm{~mm} \mathrm{Hg}$ in the right eye, 

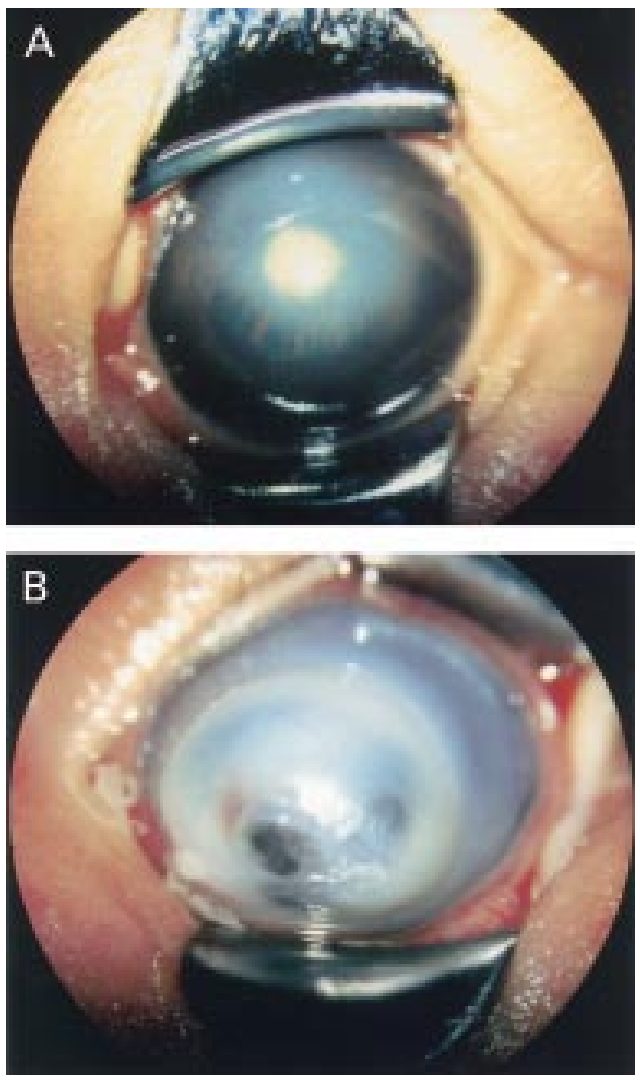

Figure 1 (A) A photograph of anterior segment of case 1. Central corneal opacity, elongated ciliary processes, and whitish mass behind the lens are observed. (B) $A$ photograph of anterior segment of case 2. The cornea is noticeably protruding and densely opaque overall. These findings correspond to anterior staphyloma.

and $20 \mathrm{~mm} \mathrm{Hg}$ in the left. The corneal diameter was $10.0 \mathrm{~mm} \times 9.5 \mathrm{~mm}$ in the right eye, and $11.0 \mathrm{~mm} \times 10.5 \mathrm{~mm}$ in the left. The axial length was $16.2 \mathrm{~mm}$ in the right eye, and $17.8 \mathrm{~mm}$ in the left. Clinically, the patient was diagnosed as having Peters' anomaly and anterior PHPV. He was referred to a paediatrician for an examination of associated systemic anomalies; however, none was detected. Chromosomal analysis showed normal karyotype, and all serological test results for TORCH embryopathy were negative. In addition, we examined the parents of the patient, and they showed no ophthalmological anomalies. The IOP in the patient's left eye elevated to $30 \mathrm{~mm}$ $\mathrm{Hg}$ or more from 3 weeks after birth. Medical treatment could not control the IOP in the left eye, and cyclocryocautery was performed on the left eye at 8 weeks after birth. However, the IOP in the left eye still could not be controlled, and the left eye manifested anterior staphyloma. Since the patient could not close his left eye because of severely progressing anterior staphyloma, his left eye was enucleated at age 7 months and underwent histopathological examination.

Case 2

A 6 week old boy was referred to our clinic with corneal opacity in the left eye that had been present since birth. At the first examination, central corneal opacity and a poorly formed anterior chamber were seen in the left eye. The
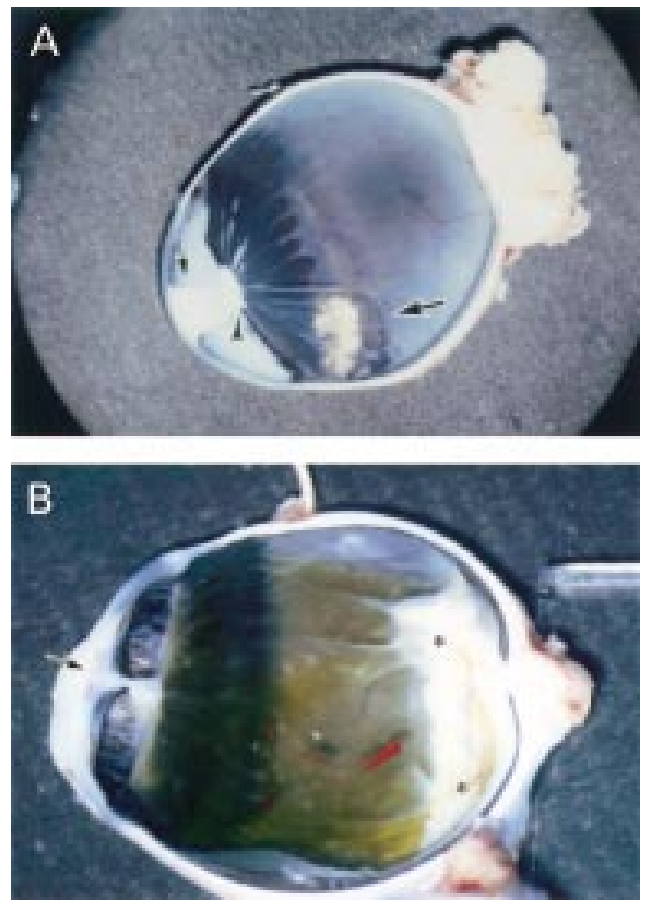

Figure 2 (A) Photograph of the section of the eyeball of case 1. The degenerative lens (small arrow) adheres to the defective central cornea, the whitish mass (arrowhead) is observed behind the lens, and ciliary coloboma is seen (large arrow). Elongated ciliary processes are also observed.

(B) Photograph of the section of the eyeball of case 2 . The degenerative lens (arrow) adheres to the defective central cornea, and the whitish membranous tissue (asterisks) is observed on the posterior retina.

cornea protruded anteriorly, corresponding to anterior staphyloma (Fig 1B). The fundus could not be observed in the left eye. No pathological findings were seen in the right eye. The IOP was $21 \mathrm{~mm} \mathrm{Hg}$ in the right and impossible to measure in the left due to the severely protruded cornea. The corneal diameter was $10.0 \mathrm{~mm} \times 10.5 \mathrm{~mm}$ in the right eye, and $14.0 \mathrm{~mm} \times 12.0 \mathrm{~mm}$ in the left. The patient was diagnosed as having Peters' anomaly. He was referred to a paediatrician for an examination of associated systemic anomalies; however, none was detected. Chromosomal analysis showed normal karyotype, and all serological test results for TORCH embryopathy were negative. We examined the parents of the patient, and they showed no ophthalmological anomalies. Since the patient could not close his left eye because of severely progressing anterior staphyloma, and the protruding thin cornea seemed to perforate, the left eye was enucleated at age 4 months and underwent histopathological examination.

HISTOPATHOLOGICAL PROCEDURES

The enucleated eyes were fixed immediately in $4 \%$ paraformaldehyde solution at $20^{\circ} \mathrm{C}$. Following fixation for approximately 7 days, the fixed tissue blocks were dehydrated in a series of ascending concentrations of ethanol, cleared in xylene, and embedded in paraffin wax. Serial sections of the eyes were cut at a thickness of $4.0 \mu \mathrm{m}$ and mounted on glass slides. After being dewaxed in xylene, sections were hydrated in a series of descending concentrations of ethanol. The hydrated sections were stained 

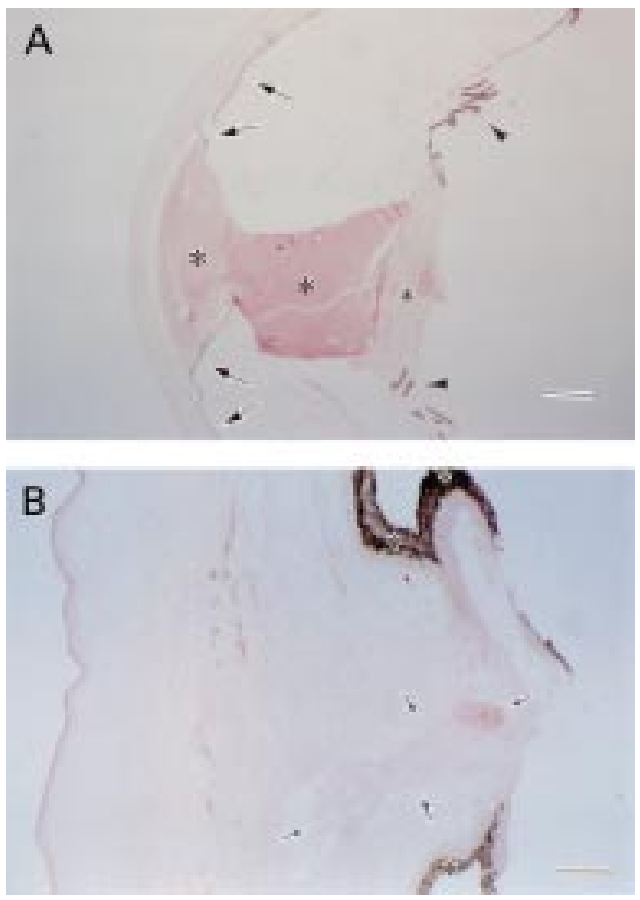

Figure 3 (A). A histological photograph of the anterior segment of case 1. The degenerative lens (large asterisks) adheres to the posterior surface of the defective corneal stroma. The anterior chamber space is not formed, and the undifferentiated iris stroma (arrows) adheres to the posterior surface of the peripheral cornea and lens.

Mesenchymal tissue (small asterisk) is observed behind the degenerative lens. Elongated ciliary processes (arrowheads) are also observed. Haematoxylin and eosin. Bar $=700 \mu \mathrm{m}$. (B). A histological photograph of the anterior segment of case 2. In the central cornea, the defects in the endothelium, Descemet's membrane, and posterior stroma are observed, and the lens material (arrows) is detected in the defective corneal stroma. The anterior chamber space is not formed, and the undifferentiated iris stroma (asterisks) adheres to the posterior surface of the peripheral cornea. Haematoxylin and eosin. Bar $=280 \mu \mathrm{m}$.

with Meyer's haematoxylin solution at $20^{\circ} \mathrm{C}$ for 10 minutes, rinsed in tap water for $15 \mathrm{~min}-$ utes, immersed in $0.5 \%$ eosin solution at $20^{\circ} \mathrm{C}$ for 10 minutes, dehydrated in a series of ascending concentrations of ethanol, cleared in xylene, and mounted in Harleco synthetic resin solution (Kokusai Shiyaku, Japan). All the sections were examined by light microscopy using Provis AX 70 with U-photo (Olympus Co, Japan) and were recorded by photomicrographs.

\section{Results}

In both eyes, the defects in the endothelium, Descemet's membrane, and posterior stroma were observed in the central cornea (Figs 2A, $2 \mathrm{~B}, 3 \mathrm{~A}, 3 \mathrm{~B}, 4 \mathrm{~A})$. The degenerative lens adhered to the posterior surface of the defective corneal stroma in both eyes (Figs 2A, $2 \mathrm{~B}, 3 \mathrm{~A}, 3 \mathrm{~B}$ ), and lens material was detected in the corneal stroma of case 2 (Fig 3B). In both eyes, the anterior chamber space was not formed, and the undifferentiated iris stroma adhered to the posterior surface of the peripheral cornea and the lens (Figs 3A, 3B). The iris stroma, ciliary body stroma, and trabecular meshwork were poorly differentiated, and Schlemm's canal was not observed in either eye (Fig 4B). The neural retina was fairly well differentiated in both eyes. Mesenchymal tissue
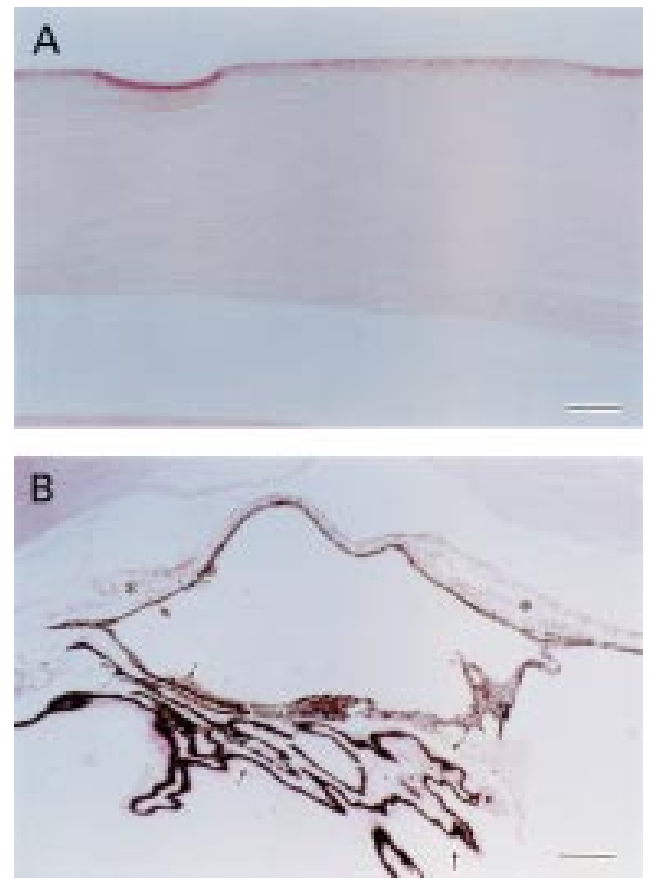

Figure 4 (A) A histological photograph of the central cornea of case 1. In the central cornea, defects in the endothelium, Descemet's membrane, and posterior stroma are observed. Haematoxylin and eosin. Bar $=70 \mu \mathrm{m}$.

(B) A histological photograph of the iris, ciliary body, and trabecular meshwork of case 2. The iris (asterisks), ciliary body (arrows), and trabecular meshwork are poorly differentiated, and Schlemm's canal is not observed. Haematoxylin and eosin. Bar $=175 \mu \mathrm{m}$.
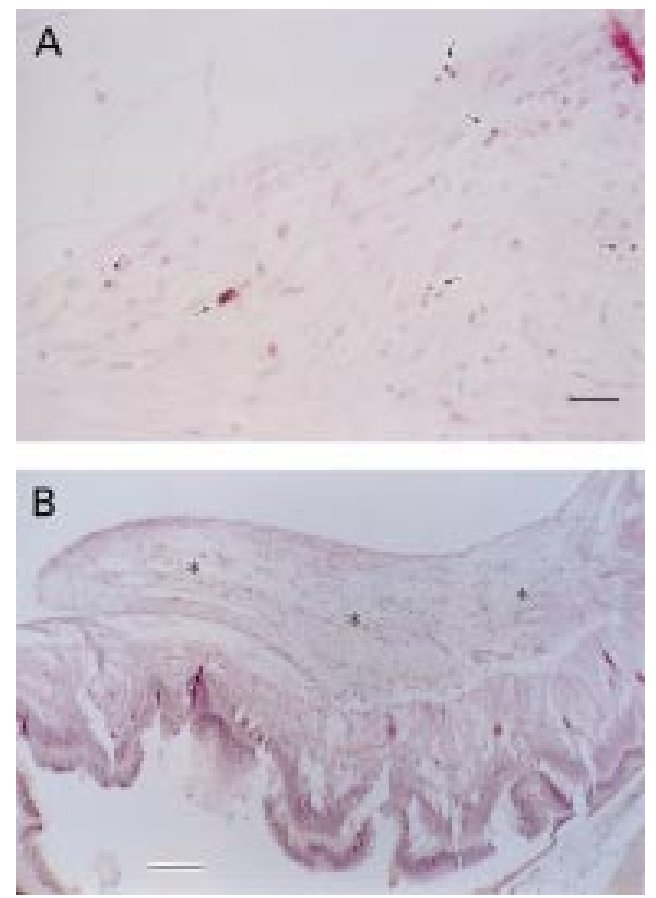

Figure 5 (A) A histological photograph of the mesenchymal tissue behind the lens of case 1. A few melanocytes (arrows) are observed in the mesenchymal tissue. Haematoxylin and eosin. Bar $=28 \mu \mathrm{m}$. (B) $A$ histological photograph of the mesenchymal tissue on the retina of case 2. Mesenchymal tissue (asterisks) containing scattered melanocytes in the vitreous cavity are seen on the retina, inducing folds in the neural retina. Haematoxylin and eosin. Bar $=280 \mu \mathrm{m}$. 
containing melanocytes was observed behind the degenerative lens (Figs 2A, 3A, 5A), and the pigment epithelium was absent at the lower nasal side of the ciliary body (Fig 2A) in case 1 . In case 2 , mesenchymal tissue containing scattered melanocytes in the vitreous cavity was seen on the posterior retina, inducing folds in the neural retina (Fig 5B). According to these histological findings, both cases were diagnosed as Peters' anomaly, PHPV, maldevelopment of the iris and ciliary body, and goniodysgenesis. In addition, case 1 had typical ciliary coloboma.

\section{Discussion}

Both eyes exhibited Peters' anomaly, PHPV, maldevelopment of the iris and ciliary body, and goniodysgenesis. In addition, case 1 had typical ciliary coloboma.

According to the classification for PHPV reported by Pruett and Schepens, ${ }^{11}$ case 1 with the retrolental mesenchymal tissue corresponds to the anterior type, while case 2 with mesenchymal tissue on the retinal surface correlates with the posterior type.

We evaluated histological findings embryologically. Waring and Rodrigues ${ }^{16}$ indicated various developmental mechanisms for Peters' anomaly, including faulty separation of the lens vesicle from the surface ectoderm, primary abnormal migration of neural crest cells into the cornea, and intrauterine corneal inflammation. In both eyes in this study, the undifferentiated iris adhered to the posterior surface of the peripheral cornea and the lens. In addition, lens material was detected in the corneal stroma of case 2 . These histopathological findings indicated that Peters' anomaly in both eyes was caused by faulty separation of the lens vesicle from the surface ectoderm.

Then, we inferred a developmental mechanism for the clinically uncommon complex of Peters' anomaly, PHPV, maldevelopment of the iris and ciliary body, goniodysgenesis, and typical coloboma. The corneal endothelium and stroma have been demonstrated to be of cranial neural crest origin, ${ }^{17-19}$ and Peters' anomaly has been attributed to mesenchymal dysgenesis of the anterior ocular segments resulting from the abnormal development of neural crest cells, as well as posterior embryotoxon and Axenfeld-Rieger syndrome. ${ }^{2-4}$

The mesenchymal PHPV tissues in both eyes contained melanocytes. As demonstrated by Shirai $^{20}$ in a experimental study using ochratoxin A as a teratogen, this pathological observation indicates that the mesenchymal PHPV tissue in both cases is derived from neural crest cells, because melanocytes have been shown to be of neural crest origin. ${ }^{321}$ Moreover, we previously demonstrated in mice, using experimental teratological methods, that teratogen induced developmental abnormalities of the vitreous, corresponding to PHPV, are caused by the abnormal migration of excessive mesenchymal cells derived from the neural crest and occasionally accompanied by faulty separation of the lens vesicle, corresponding to Peters' anomaly. ${ }^{20} 22$
In both cases, maldevelopment of the iris stroma, ciliary stroma, and goniodysgenesis were observed. The iris stroma, ciliary stroma, and trabecular meshwork have been demonstrated to be derived from neural crest cells. ${ }^{17-19}$ Therefore, we suggest that developmental disorders of neural crest cells are responsible for maldevelopment of the iris stroma, ciliary stroma, and goniodysgenesis, as observed in both of these cases. In addition, it was demonstrated in mice, using experimental teratological methods, that teratogen induced faulty separation of the lens vesicle corresponding to Peters' anomaly, often accompanies iridocorneal malformations as our cases. ${ }^{23}{ }^{24}$ Based on these experimental data, it seems that Peters' anomaly accompanied maldevelopment of the iris stroma, ciliary stroma, and goniodysgenesis in both cases.

Typical ciliary coloboma was observed in case 1. We previously demonstrated in mice, using experimental teratological methods, that faulty closure of the embryonic fissure, corresponding to typical uveal coloboma, is caused by the abnormal migration of excessive mesenchymal cells derived from the neural crest. ${ }^{20}$ Moreover, we recently reviewed 72 cases with typical uveal coloboma, and demonstrated that most of the associated ocular and systemic anomalies were detected in the tissues derived from neural crest cells, and concluded clinically that developmental disorders of neural crest cells may be related to typical uveal coloboma. ${ }^{26}$

As described above, we inferred that migratory disorders of neural crest cells are responsible for Peters' anomaly, PHPV, maldevelopment of the iris and ciliary body, goniodysgenesis, and typical coloboma.

Finally, we inferred a critical period for the complex of Peters' anomaly, PHPV, maldevelopment of the iris and ciliary body, goniodysgenesis, and typical coloboma. Embryologically, in normal human eye development, it has been demonstrated that the lens vesicle separates from surface ectoderm, cranial neural crest cells migrate into the anterior segments and vitreous cavity, and the embryonic fissure closes at from 4 to 7 weeks of gestation. ${ }^{20}{ }^{27}$ As we described here, abnormal development of neural crest cells during this period is responsible for Peters' anomaly, PHPV, maldevelopment of the iris and ciliary body, goniodysgenesis, and typical coloboma.

Although IOP in the left eye of case 2 was impossible to measure, anterior staphyloma progressed rapidly in both cases. We inferred that in addition to increased IOP due to the undifferentiated chamber angle, maldevelopment of the cornea resulted in severely progressing anterior staphyloma. Patients with Peters' anomaly should be checked the development of severe anterior staphyloma even when they have a normal IOP.

Based on the findings of this study, we present a hypothesis on the mechanism and critical period for the complex of Peters' anomaly, PHPV, maldevelopment of the iris and ciliary body, goniodysgenesis, and typical coloboma, which states that migratory disorders of neural crest cells from 4 to 7 weeks of 
gestation are responsible for the malformation complex in both cases.

1 Peters A. Uber angeborene Defektbildung der Descemetschen Membran. Klin Monatsbl Augenheilkd 1906;44:2740 .

2 Bahn CF, Falls HF, Varley GA, et al. Classification of corneal endothelial disorders based on neural crest origin. Ophthalmology 1984;91:558-63.

3 Beauchamp GR, Knepper PA. Role of the neural crest in anterior segment development and disease. I Pediatr Ophthalmol Strabismus 1984;21:209-14.

4 Waring GO. Congenital and neonatal corneal abnormalities. In: Leibowitz HW, ed. Corneal disorders. Clinical diagnosi and management. Philadelphia: Saunders, 1984:29-56.

5 Van Schooneveld MJ, Delleman JW, Beemer FA, et al. Peters'-plus: a new syndrome. Ophthalmic Paediatr Genet 1984;4:141-6.

6 Heon E, Barsoum-Homsy M, Cevrette L, et al. Peters' anomaly. Ophthalmic Paediatr Genet 1992;13:137-43.

7 Mayer UM. Peters' anomaly and combination with other malformations. Ophthalmic Paediatr Genet 1992;13:131-5.

8 Traboulsi EI, Maumenee IH. Peters' anomaly and associated congenital malformations. Arch Ophthalmol 1992;110: ated congenit.

9 Myles WM, Flanders ME, Chitayat D, et al. Peters' anomaly: a clinicopathologic study. $\mathcal{F}$ Pediatr Ophthalmo Strabismus 1992;29:374-81.

10 Reese AB. Persistent hyperplastic primary vitreous. Am $\mathcal{f}$ Ophthalmol 1955;40:317-31.

11 Pruett RC, Schepens CL. Posterior hyperplastic primary vitreous. Am $\mathcal{F}$ Ophthalmol 1970;69:535-43.

12 Mann I. Congenital retinal fold. Br f Ophthalmol 1935;19: 641-58.

13 Weve H. Uber Ablatio falciformis congenita. Arch Augenheilkd 1935;109:371-94.

14 Haddad R, Font RL, Reeser F. Persistent hyperplastic primary vitreous. A clinicopathologic study of 62 cases and review of the literature. Surv Ophthalmol 1978;23:123-34.
15 Uemura A. Clinico-anatomical studies on developing vitreous and retina. F Fpn Ophthalmol Soc 1986;90:1-24.

16 Waring GO, Rodrigues MM. Congenital and neonatal corneal abnormalities. In: Tasman W, Jaeger EA, ed. Foundations of clinical ophthalmology. Vol 1, Chap 9. Philadelphia: Lippincott, 1993:1-38.

17 Johnston MC, Noden DM, Hazelton RD, et al. Origins of avian ocular and periocular tissues. Exp Eye Res 1979;29: $27-43$.

18 Laibson PR, Waring GO. Diseases of the cornea. In: Harley RD, ed. Pediatric ophthalmology. 2nd ed. Philadelphia; Saunders, 1983:456-514.

19 Cook CS, Ozanics V, Jakobiec FA. Prenatal development of the eye and its adnexa. In: Tasman W, Jaeger EA, ed. Foundations of clinical ophthalmology. Vol 1, Chap 2. Philadelphia: Lippincott, 1991:1-93.

20 Shirai S. Developmental mechanisms of congenital eye abnormalities. F fpn Ophthalmol Soc 1991;95:1206-37.

21 Noden DM. Periocular mesenchyme. Neural crest and mesodermal interactions. In: Tasman W, Jaeger EA, ed. mesodermal interactions. In: Tasman W, Jaeger EA, ed.
Foundations of clinical ophthalmology. Vol 1, Chap 3. Foundations of clinical ophthalmology.

22 Ozeki H, Shirai S, Ikeda K, et al. Critical period for retinoic acid-induced developmental abnormalities of the vitreous in mouse fetuses. Exp Eye Res 1999;68:223-8.

23 Cook CS. Sulik KK. Keratolenticular dysgenesis (Peters' anomaly) as a result of acute embryonic insult during gastrulation. F Pediatr Ophthalmol Strabismus 1988;25:60-6.

24 Cook CS. Experimental models of anterior segment dysgenesis. Ophthalmic Paediatr Genet 1989;10:33-46.

25 Ozeki H, Shirai S. Developmental eye abnormalities in mouse fetuses induced by retinoic acid. Fpn f Ophthalmol 1998;42:162-7.

26 Ozeki H, Shirai S, Nozaki M, et al. Maldevelopment of neural crest cells in patients with typical uveal coloboma. $\mathcal{F}$ Pediatr Ophthalmol Strabismus 1999;36:337-41.

27 Kameyama Y. Comparative developmental pathology of congenital malformations of the central nervous system. Brain Development 1981;3:450-9. 